\title{
Genetic diversity of standard leaf nutrients in Coffea canephora genotypes during phenological phases
}

\author{
W.R. Gomes ${ }^{1}$, W.P. Rodrigues ${ }^{2}$, H.D. Vieira ${ }^{2}$, M.G. Oliveira ${ }^{1}$, J.R.M. Dias ${ }^{3}$ \\ and F.L. Partelli ${ }^{1}$ \\ ${ }^{1}$ Centro Universitário Norte do Espírito Santo, \\ Universidade Federal do Espírito Santo, São Mateus, ES, Brasil \\ ${ }^{2}$ Centro de Ciências e Tecnologias Agropecuárias, \\ Universidade Estadual do Norte Fluminense, Campos dos Goytacazes, RJ, Brasil \\ ${ }^{3}$ Universidade Federal de Rondônia, Rolim de Moura, RO, Brasil \\ Corresponding author: F.L. Partelli \\ E-mail: partelli@yahoo.com.br
}

Genet. Mol. Res. 15 (4): gmr.15048839

Received May 31, 2016

Accepted July 12, 2016

Published October 6, 2016

DOI http://dx.doi.org/10.4238/gmr.15048839

Copyright $(2016$ The Authors. This is an open-access article distributed under the terms of the Creative Commons Attribution ShareAlike (CC BY-SA) 4.0 License.

\begin{abstract}
Diagnosing foliar nutritional status is essential for fertilizer recommendations and for the identification of nutrient imbalances. This study aimed to verify genetic diversity and establish mean standards (leaf nutrient contents; LNCs) and relationships among leaf nutrients (LNC relationships; LNCRs) in seven conilon coffee genotypes during both pre-flowering and bean-filling stages. Twenty crops from several cities in the northern region of Espírito Santo State, Brazil, with crop yield either equal to or greater than 100 bags per hectare (during two harvests) were assessed. A total of 140 samples were collected during each evaluation period for quantification of leaf nutrient contents ( $\mathrm{N}, \mathrm{P}, \mathrm{K}, \mathrm{Ca}, \mathrm{Mg}, \mathrm{S}, \mathrm{Fe}, \mathrm{Zn}, \mathrm{Cu}, \mathrm{Mn}$, and $\mathrm{B})$. The
\end{abstract}


Ward procedure, a hierarchical genetic clustering method, was used to quantify the genetic diversity among genotypes. To examine differences between the LNCs and LNCRs, F-and Scott-Knott tests were used. LNCs and LNCRs showed significant differences among the conilon coffee genotypes during the evaluation periods. Additionally, the $8 \mathrm{~V}$, $10 \mathrm{~V}$, and $12 \mathrm{~V}$ genotypes exhibited the highest values for most of the nutrients, especially for $\mathrm{N}, \mathrm{P}$, and $\mathrm{Cu}$. Therefore, the clustering method revealed genetic diversity among genotypes for standard leaf nutrient levels, implying that leaf diagnosis could be specific to each genotype and phenological stage.

Key words: Conilon coffee; Sampling time; Mineral nutrition; Mean leaf nutrient content; Cluster

\section{INTRODUCTION}

Brazil, which stands out as the largest global producer and exporter and the second largest consumer of coffee beans, has the most extensive crop cultivation area in the world (ICO, 2016). Among the 124 Coffea species, only Coffea arabica L. and Coffea canephora Pierre ex A. Froehner, commercially known as arabica and robusta/conilon coffee, respectively, have economic importance worldwide (Davis et al., 2011, 2012). World coffee production exceeded 143 million bags in 2015, of which approximately 59 million bags were derived from $C$. canephora (ICO, 2016), and Brazil maintained its prominent position with the production of approximately 11 million bags from this species (CONAB, 2016).

The use of new technologies, such as improved varieties, mechanization, irrigation, correct use of liming and fertilization, leaf analysis, programmed pruning cycles, densification and phytosanitary controls, has significantly contributed to the increase in conilon coffee yield (Partelli et al., 2006, 2014a; Covre et al., 2015). However, there is limited information regarding the specific mineral nutrient composition of conilon coffee genotypes, particularly standard leaf nutrient levels for a specific diagnosis, taking into account genotype and phenological stages.

Research focused on breeding conilon coffee has resulted in the generation of new clonal varieties (Fonseca et al., 2004, 2006). However, phenotypic differences such as morphological, physiological, and yield traits among $C$. canephora clones indicate that genetic variability exists in terms of production and fruit ripening time (Fonseca et al., 2006), as well as in patterns of nutrient absorption and allocation among conilon genotypes (Covre et al., 2013). This is probably due to the cross pollination reproduction mode of this species.

The existence of genetic variability within C. canephora germplasm (Fonseca et al., 2004), due to the allogamous reproduction of this species, results in genotypes with different phenotypic traits (Esther and Adomako, 2010; Leroy et al., 2014). Because commercial conilon coffee crops are explored using clonal varieties (different genotypes grown in the same area) to ensure satisfactory pollination, phenotypic traits, if correctly observed, can help to identify different management options. These include different phytosanitary control methods (Belan et al., 2015) and different fertilization practices for each genotype, which optimize yield costs.

Studies investigating the growth and development of conilon coffee have revealed genetic variability in leaf number, plant height, emission rate of reproductive branches, root and shoot dry matter, and nutrient accumulation (Contarato et al., 2010; Covre et al., 2013;

Genetics and Molecular Research 15 (4): gmr.15048839 
Partelli et al., 2014b; Marré et al., 2015; Ronchi et al., 2015). Additionally, some studies have shown variation related to nutrient-use efficiency among conilon genotypes (Favarin et al., 2007; Tomaz et al., 2009; Ferreira et al., 2013) and different nutritional requirements according to fruit ripeness stage and time of the year (Marré et al., 2015). In this context, the use of tools that can identify genetic diversity, such as hierarchical genetic clustering methods (multivariate methods), can aid in the identification of $C$. canephora genotypes with different genetic distances (Kathurima et al., 2012; Souza et al., 2013; Dalcomo et al., 2015; Martins et al., 2015). Among the multivariate methods, the Ward method provides an excellent strategy for quantifying genetic diversity. The nutritional status of conilon coffee leaves has been interpreted using the "sufficiency range" method, which generates results that are easy to understand (Partelli et al., 2006, 2007). The standard values from this method are usually regional and a mean value of the leaf nutrient content (LNC) is used as a reference (Partelli et al., 2006) according to the time of year (Partelli et al., 2007); however, it does not take into account genetic diversity. Additional, standard values for specific genotypes have not been established. Consequently, the objective of the present study was to verify the genetic diversity of leaf nutrients and establish the LNCs and LNC relationships (LNCRs) of leaves from seven conilon coffee genotypes during two phenological stages (pre-flowering and bean filling).

\section{MATERIAL AND METHODS}

This study was performed on commercial conilon coffee crops (C. canephora Pierre ex A. Froehner) scattered across the northern region of Espírito Santo State, Brazil. The climate is classified as Aw, i.e., a warm tropical climate with a dry season during winter and a rainy season during summer according to the Köppen classification (Köppen, 1931). Twenty crops from Vila Valério, Jaguaré, Nova Venécia, São Mateus, São Gabriel da Palha, Boa Esperança, Vila Pavão, São Domingos do Norte, Águia Branca, and Governador Lindenberg cities, with crop yields equal to or greater than 100 bags per hectare (average from harvests conducted in 2012 and 2013) were used. These conilon coffee crops were managed using irrigation, fertilization, liming, and pest control, and each clone was planted separately in lines, with the number of plants ranging from 2777 to 3570 per hectare.

Leaf samples were collected in 2012 from seven conilon genotypes belonging to the clonal variety Incaper Vitória 8142 (Fonseca et al., 2004), during the pre-flowering (between late May and early June) and bean-filling (late November and early December) stages; a composite sampleof100 leaves was collected during each evaluation period. The third and/or fourth pair of mature leaves from the plagiotropic branch apex located in the upper third of the plant was placed in a paper bag and transported to the laboratory to determine the leaf $\mathrm{N}, \mathrm{P}, \mathrm{K}, \mathrm{Ca}, \mathrm{Mg}, \mathrm{S}, \mathrm{Fe}, \mathrm{Zn}, \mathrm{Cu}$, and $\mathrm{Mn}$ nutrient contents according to the method described by Silva (2009). We subsequently constructed a database to obtain the mean values (LNCs) for each genotype and bivariate relationships (LNCRs) obtained in direct and inverse forms, among all the nutrients studied.

The data were analyzed simultaneously using the Ward method to create groups through clustering. For the Ward clustering method, the distance matrix was constructed by Mahalanobis's algorithm (Mahalanobis, 1936) using the Genes statistical software (Cruz, 2013). The distance matrix was used as a dissimilarity measure for the clustering analysis of the genotypes by the Ward method (dendrogram) using the statistical $\mathrm{R}$ software. To validate the clustering analysis, or to check the dendrogram's ability to play the dissimilarity matrices (D and $\mathrm{D}^{2}$ ), we calculated the cophenetic correlation coefficient (CCC). The CCC is the Pearson

Genetics and Molecular Research 15 (4): gmr.15048839 
correlation coefficient between the matrices of distance (D and $\left.\mathrm{D}^{2}\right)$ and cophenetic matrix $(\mathrm{C})$ (matrix of distances between genotypes, obtained from the dendrogram), and values close to unity indicate better representation (Cruz et al., 2014). LNC and LNCR data during both preflowering and bean filling stages were subjected to analysis of variance, and the means were grouped according to the Scott-Knott test at 5\% probability using the Assistant version 7.6 beta program (Silva and Azevedo, 2002).

\section{RESULTS AND DISCUSSION}

Based on Mahalanobis distance $\left(\mathrm{D}^{2}\right)$, two clusters (Figure 1) were formed considering the cut-off point of the dendrogram proposed by Mojena (1977). The first cluster was formed by $6 \mathrm{~V}, 13 \mathrm{~V}, 9 \mathrm{~V}$, and $5 \mathrm{~V}$, and the second cluster was formed by $8 \mathrm{~V}, 10 \mathrm{~V}$, and $12 \mathrm{~V}$ genotypes, which showed higher LNCs. Using the Mantel test $(\mathrm{P}<0.01)$ with 1000 permutations, we confirmed the association between the cophenetic matrix and the distance matrix $\left(\mathrm{D}^{2}\right)$, with the CCC being higher than $65 \%$. (Figure 1). Although two clusters were formed, the presentation of individual LNC and LNCR values is useful since they can be used as reference values for each genotype.

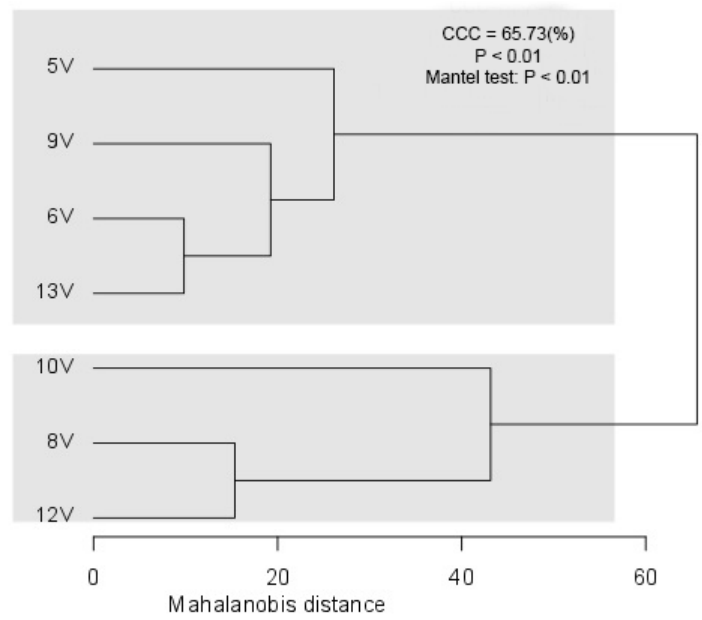

Figure 1. Dendrogram obtained by the Ward hierarchical method based on Mahalanobis distance of seven Coffea canephora $\mathrm{cv}$. belonging to the clonal variety Incaper Vitória 8142 with the cut-off point determined according to Mojena (1977) and cophenetic correlation coefficient (CCC).

There were significant differences among conilon coffee genotypes for LNC and LNCR during both the pre-flowering and bean-filling stages (Tables 1, 2, 3, and 4). These results indicate the formation of two groups of different composition depending on the genotypes for most of the nutrients analyzed. However, for $\mathrm{Ca}$ and $\mathrm{Zn}$, and $\mathrm{S}$, a third and fourth group were formed, respectively. During the pre-flowering stage, $\mathrm{K}, \mathrm{Mg}, \mathrm{B}, \mathrm{Fe}$, and $\mathrm{Mn}$ were statistically similar among all analyzed coffee genotypes (Table 1). Conversely, the $8 \mathrm{~V}, 9 \mathrm{~V}$, and $12 \mathrm{~V}$ genotypes exhibited the highest values for most of the nutrients, especially for $\mathrm{N}, \mathrm{P}$, and $\mathrm{Cu}$. Three groups were formed for $\mathrm{Ca}$ and $\mathrm{Zn}$, among which the $10 \mathrm{~V}$ and $12 \mathrm{~V}$ genotypes, respectively, had the highest mean values. Conversely, the $5 \mathrm{~V}, 6 \mathrm{~V}$, and $10 \mathrm{~V}$ genotypes were in the group with the lowest $\mathrm{S}$ contents.

Genetics and Molecular Research 15 (4): gmr.15048839 
Table 1. Mean values, coefficient of variation (CV), and F- and Scott-Knott test results for nutrient contents measured in seven conilon genotypes of the clonal variety Vitória Incaper 8142 with crop yield either equal to or greater than 100 bags per hectare (average from 2012 and 2013 harvests) during the pre-flowering stage.

\begin{tabular}{|c|c|c|c|c|c|c|c|c|c|}
\hline \multirow[t]{2}{*}{ Nutrients } & \multicolumn{7}{|c|}{ Genotypes } & \multirow[t]{2}{*}{$\mathrm{CV}$} & \multirow[t]{2}{*}{ F test } \\
\hline & $5 \mathrm{~V}$ & $6 \mathrm{~V}$ & $8 \mathrm{~V}$ & $9 \mathrm{~V}$ & $10 \mathrm{~V}$ & $12 \mathrm{~V}$ & $13 \mathrm{~V}$ & & \\
\hline $\mathrm{N}(\mathrm{g} / \mathrm{kg})$ & $24.2^{\mathrm{b}}$ & $25.5^{\mathrm{b}}$ & $26.6^{\mathrm{a}}$ & $26.9^{\mathrm{a}}$ & $25.1^{\mathrm{b}}$ & $28.1^{\mathrm{a}}$ & $24.8^{\mathrm{b}}$ & 9.70 & $* *$ \\
\hline$P(g / k g)$ & $1.9^{\mathrm{b}}$ & $1.2^{\mathrm{b}}$ & $1.3^{\mathrm{a}}$ & $1.3^{\mathrm{a}}$ & $1.2^{\mathrm{b}}$ & $1.2^{\mathrm{a}}$ & $1.3^{\mathrm{a}}$ & 16.8 & $* *$ \\
\hline $\mathrm{K}(\mathrm{g} / \mathrm{kg})$ & $11.7^{\mathrm{a}}$ & $12.4^{\mathrm{a}}$ & $13.7^{\mathrm{a}}$ & $12.2^{\mathrm{a}}$ & $12.7^{\mathrm{a}}$ & $12.3^{\mathrm{a}}$ & $12.0^{\mathrm{a}}$ & 20.2 & NS \\
\hline $\mathrm{Ca}(\mathrm{g} / \mathrm{kg})$ & $20.7^{\mathrm{b}}$ & $16.9^{\mathrm{c}}$ & $21.6^{\mathrm{b}}$ & $21.3^{\mathrm{b}}$ & $26.1^{\mathrm{a}}$ & $21.2^{\mathrm{b}}$ & $18.2^{\mathrm{c}}$ & 24.1 & $* *$ \\
\hline $\mathrm{Mg}(\mathrm{g} / \mathrm{kg})$ & $3.3^{\mathrm{a}}$ & $3.3^{\mathrm{a}}$ & $3.3^{\mathrm{a}}$ & $3.8^{\mathrm{a}}$ & $3.9^{\mathrm{a}}$ & $3.9^{\mathrm{a}}$ & $3.6^{\mathrm{a}}$ & 28.5 & NS \\
\hline $\mathrm{S}(\mathrm{g} / \mathrm{kg})$ & $1.0^{\mathrm{d}}$ & $1.3^{\mathrm{c}}$ & $1.6^{\mathrm{a}}$ & $1.4^{\mathrm{b}}$ & $1.2^{\mathrm{c}}$ & $1.7^{\mathrm{a}}$ & $1.2^{\mathrm{c}}$ & 20.0 & $* *$ \\
\hline $\mathrm{B}(\mathrm{mg} / \mathrm{kg})$ & $76.8^{\mathrm{a}}$ & $60.9^{\mathrm{a}}$ & $75.2^{\mathrm{a}}$ & $79.6^{\mathrm{a}}$ & $77.2^{\mathrm{a}}$ & $83.5^{\mathrm{a}}$ & $71.4^{\mathrm{a}}$ & 31.7 & NS \\
\hline $\mathrm{Cu}(\mathrm{mg} / \mathrm{kg})$ & $7.9^{\mathrm{b}}$ & $8.1^{\mathrm{b}}$ & $11.4^{\mathrm{a}}$ & $9.4^{\mathrm{a}}$ & $7.1^{\mathrm{b}}$ & $11.0^{\mathrm{a}}$ & $10.9^{\mathrm{a}}$ & 52.1 & $*$ \\
\hline $\mathrm{Fe}(\mathrm{mg} / \mathrm{kg})$ & $149.1^{\mathrm{a}}$ & $118.4^{\mathrm{a}}$ & $129.9^{\mathrm{a}}$ & $130.6^{\mathrm{a}}$ & $131.3^{\mathrm{a}}$ & $145.1^{\mathrm{a}}$ & $113.5^{\mathrm{a}}$ & 49.1 & NS \\
\hline $\mathrm{Mn}(\mathrm{mg} / \mathrm{kg})$ & $139.9^{\mathrm{a}}$ & $150.9^{a}$ & $144.7^{\mathrm{a}}$ & $136.3^{\mathrm{a}}$ & $153.4^{\mathrm{a}}$ & $135.1^{\mathrm{a}}$ & $147.8^{\mathrm{a}}$ & 57.7 & NS \\
\hline $\mathrm{Zn}(\mathrm{mg} / \mathrm{kg})$ & $5.4^{\mathrm{c}}$ & $6.4^{\mathrm{c}}$ & $7.0^{\mathrm{b}}$ & $5.9^{\mathrm{c}}$ & $6.2^{\mathrm{c}}$ & $8.2^{\mathrm{a}}$ & $5.8^{\mathrm{c}}$ & 21.2 & ** \\
\hline
\end{tabular}

For each nutrient, mean values followed by different letters indicate significant differences between genotypes according to the Scott-Knott test at 5\% probability. NS = not significant; **highly significant $(\mathrm{P} \leq 0.01)$; ${ }^{*}$ significant $(\mathrm{P} \leq 0.05)$.

Table 2. Mean values, CV, and F- and Scott-Knott test results for nutrient contents measured in seven conilon genotypes belonging to the clonal variety Vitória Incaper 8142 with crop yield either equal to or greater than 100 bags per hectare (average from 2012 and 2013 harvests) during the bean-filling stage.

\begin{tabular}{|c|c|c|c|c|c|c|c|c|c|}
\hline \multirow[t]{2}{*}{ Nutrients } & \multicolumn{7}{|c|}{ Genotypes } & \multirow[t]{2}{*}{$\mathrm{CV}$} & \multirow[t]{2}{*}{ F test } \\
\hline & $5 \mathrm{~V}$ & $6 \mathrm{~V}$ & $8 \mathrm{~V}$ & $9 \mathrm{~V}$ & $10 \mathrm{~V}$ & $12 \mathrm{~V}$ & $13 \mathrm{~V}$ & & \\
\hline $\mathrm{N}(\mathrm{g} / \mathrm{kg})$ & $26.3^{\mathrm{b}}$ & $27.0^{\mathrm{b}}$ & $28.8^{\mathrm{a}}$ & $29.1^{\mathrm{a}}$ & $26.6^{\mathrm{b}}$ & $29.9^{\mathrm{a}}$ & $27.8^{\mathrm{b}}$ & 8.7 & $* *$ \\
\hline $\mathrm{P}(\mathrm{g} / \mathrm{kg})$ & $1.2^{\mathrm{b}}$ & $1.3^{\mathrm{b}}$ & $1.4^{\mathrm{a}}$ & $1.4^{\mathrm{a}}$ & $1.2^{\mathrm{b}}$ & $1.3^{\mathrm{b}}$ & $1.37^{\mathrm{a}}$ & 15.3 & $* *$ \\
\hline $\mathrm{K}(\mathrm{g} / \mathrm{kg})$ & $15.9^{\mathrm{a}}$ & $15.7^{\mathrm{a}}$ & $16.9^{\mathrm{a}}$ & $15.9^{\mathrm{a}}$ & $16.4^{\mathrm{a}}$ & $14.7^{\mathrm{a}}$ & $15.8^{\mathrm{a}}$ & 18.3 & NS \\
\hline $\mathrm{Ca}(\mathrm{g} / \mathrm{kg})$ & $15.2^{\mathrm{c}}$ & $14.7^{\mathrm{c}}$ & $21.2^{\mathrm{a}}$ & $17.8^{\mathrm{b}}$ & $22.3^{\mathrm{a}}$ & $20.9^{\mathrm{a}}$ & $15.3^{\mathrm{c}}$ & 17.8 & $* *$ \\
\hline $\mathrm{Mg}(\mathrm{g} / \mathrm{kg})$ & $2.6^{\mathrm{c}}$ & $3.3^{\mathrm{b}}$ & $3.7^{\mathrm{a}}$ & $3.3^{\mathrm{b}}$ & $3.5^{\mathrm{a}}$ & $3.7^{\mathrm{a}}$ & $3.2^{\mathrm{b}}$ & 21.6 & $* *$ \\
\hline $\mathrm{S}(\mathrm{g} / \mathrm{kg})$ & $1.1^{\mathrm{d}}$ & $1.3^{\mathrm{c}}$ & $1.8^{\mathrm{a}}$ & $1.6^{\mathrm{b}}$ & $1.1^{\mathrm{d}}$ & $1.9^{\mathrm{a}}$ & $1.4^{\mathrm{c}}$ & 18.4 & $* *$ \\
\hline $\mathrm{B}(\mathrm{mg} / \mathrm{kg})$ & $78.4^{\mathrm{b}}$ & $65.3^{\mathrm{b}}$ & $86.2^{\mathrm{a}}$ & $93.2^{\mathrm{a}}$ & $77.0^{\mathrm{b}}$ & $86.1^{\mathrm{a}}$ & $73.2^{\mathrm{b}}$ & 26.2 & $* *$ \\
\hline $\mathrm{Cu}(\mathrm{mg} / \mathrm{kg})$ & $11.0^{\mathrm{a}}$ & $10.7^{\mathrm{a}}$ & $14.8^{\mathrm{a}}$ & $14.1^{\mathrm{a}}$ & $11.2^{\mathrm{a}}$ & $14.4^{\mathrm{a}}$ & $15.5^{\mathrm{a}}$ & 50.2 & $\mathrm{NS}$ \\
\hline $\mathrm{Fe}(\mathrm{mg} / \mathrm{kg})$ & $118.3^{\mathrm{a}}$ & $96.5^{\mathrm{a}}$ & $105.1^{\mathrm{a}}$ & $115.1^{\mathrm{a}}$ & $106.7^{\mathrm{a}}$ & $115.8^{\mathrm{a}}$ & $86.7^{\mathrm{a}}$ & 36.1 & $\mathrm{NS}$ \\
\hline $\mathrm{Mn}(\mathrm{mg} / \mathrm{kg})$ & $119.6^{\mathrm{a}}$ & $115.2^{\mathrm{a}}$ & $111.7^{\mathrm{a}}$ & $130.7^{\mathrm{a}}$ & $128.2^{\mathrm{a}}$ & $112.7^{\mathrm{a}}$ & $115.4^{\mathrm{a}}$ & 58.6 & NS \\
\hline $\mathrm{Zn}(\mathrm{mg} / \mathrm{kg})$ & $9.6^{\mathrm{a}}$ & $9.9^{\mathrm{a}}$ & $13.4^{\mathrm{a}}$ & $11.2^{\mathrm{a}}$ & $11.9^{\mathrm{a}}$ & $12.9^{\mathrm{a}}$ & $10.37^{\mathrm{a}}$ & 52.5 & NS \\
\hline
\end{tabular}

For each nutrient, the mean values followed by different letters indicate significant differences between genotypes according to the Scott-Knott test at $5 \%$ probability. NS = not significant; **highly significant $(\mathrm{P} \leq 0.01)$.

During the bean-filling stage (Table 2), no significant differences in $\mathrm{K}, \mathrm{Cu}$, $\mathrm{Fe}, \mathrm{Mn}$, and $\mathrm{Zn}$ were observed among the genotypes. However, there were significant differences between genotypes for at least one of the other nutrients $(\mathrm{N}, \mathrm{P}, \mathrm{Ca}, \mathrm{Mg}, \mathrm{S}$, and $\mathrm{B}$ ). Considering $\mathrm{LNC}$, the $8 \mathrm{~V}$ and $12 \mathrm{~V}$ genotypes showed similar behavior during the pre-flowering stage, with differences only in $\mathrm{P}$; the $8 \mathrm{~V}$ genotype had the highest value. Furthermore, the $5 \mathrm{~V}$ and $8 \mathrm{~V}$ genotypes were more distinct and showed significant differences in seven nutrients $(\mathrm{N}, \mathrm{P}, \mathrm{K}, \mathrm{Ca}, \mathrm{Mg}, \mathrm{S}$, and $\mathrm{B})$.

The clonal variety 'Vitória Incaper 8142' presents two clone groups during initial development (Contarato et al., 2010). In the present study, there was variability in nutrient use among the genotypes (four groups), consistent with findings from previous studies on coffee culture, in which differential use of nutrients was observed among clones of the same species (Reis Júnior and Martinez, 2002; Mattiello et al., 2008; Tomaz et al., 2009; Ferreira et al., 2013). 
Table 3. Mean values, CV, and F- and Scott-Knott test results for foliar nutrient relationships in seven conilon genotypes belonging to the clonal variety Vitória Incaper 8142 with crop yield either equal to or greater than 100 bags per hectare (average from 2012 and 2013 harvests) during the pre-flowering stage.

\begin{tabular}{|c|c|c|c|c|c|c|c|c|c|}
\hline \multirow[t]{2}{*}{ Relationships } & \multicolumn{7}{|c|}{ Genotypes } & \multirow[t]{2}{*}{$\mathrm{CV}$} & \multirow[t]{2}{*}{ F test } \\
\hline & $5 \mathrm{~V}$ & $6 \mathrm{~V}$ & $8 \mathrm{~V}$ & $9 \mathrm{~V}$ & $10 \mathrm{~V}$ & $12 \mathrm{~V}$ & $13 \mathrm{~V}$ & & \\
\hline $\mathrm{N} / \mathrm{P}$ & $22.62^{\mathrm{a}}$ & $22.13^{\mathrm{a}}$ & $20.21^{\mathrm{a}}$ & $21.30^{\mathrm{a}}$ & $22.00^{\mathrm{a}}$ & $23.10^{\mathrm{a}}$ & $20.21^{\mathrm{a}}$ & 17.0 & NS \\
\hline $\mathrm{N} / \mathrm{K}$ & $2.132^{\mathrm{a}}$ & $2.138^{\mathrm{a}}$ & $2.022^{\mathrm{a}}$ & $2.289^{\mathrm{a}}$ & $2.062^{\mathrm{a}}$ & $2.404^{\mathrm{a}}$ & $2.176^{\mathrm{a}}$ & 23.9 & NS \\
\hline$\overline{\mathrm{N} / \mathrm{Ca}}$ & $1.231^{\mathrm{b}}$ & $1.619^{\mathrm{a}}$ & $1.283^{\mathrm{b}}$ & $1.332^{\mathrm{b}}$ & $1.016^{\mathrm{c}}$ & $1.396^{\mathrm{a}}$ & $1.460^{\mathrm{a}}$ & 26.9 & $* *$ \\
\hline $\mathrm{N} / \mathrm{Mg}$ & $7.618^{\mathrm{a}}$ & $8.333^{\mathrm{a}}$ & $8.706^{\mathrm{a}}$ & $7.519^{\mathrm{a}}$ & $7.341^{\mathrm{a}}$ & $7.808^{\mathrm{a}}$ & $7.293^{\mathrm{a}}$ & 32.2 & NS \\
\hline $\mathrm{N} / \mathrm{S}$ & $23.97^{\mathrm{a}}$ & $20.81^{\mathrm{b}}$ & $17.52^{\mathrm{c}}$ & $19.56^{\mathrm{b}}$ & $20.58^{\mathrm{b}}$ & $16.87^{\mathrm{c}}$ & $20.46^{\mathrm{b}}$ & 19.7 & $* *$ \\
\hline $\mathrm{N} / \mathrm{B}$ & $0.362^{\mathrm{a}}$ & $0.484^{\mathrm{a}}$ & $0.395^{\mathrm{a}}$ & $0.379^{\mathrm{a}}$ & $0.357^{\mathrm{a}}$ & $0.382^{\mathrm{a}}$ & $0.381^{\mathrm{a}}$ & 42.4 & NS \\
\hline $\mathrm{N} / \mathrm{Cu}$ & $3.660^{\mathrm{a}}$ & $4.229^{\mathrm{a}}$ & $3.141^{\mathrm{a}}$ & $3.382^{\mathrm{a}}$ & $4.119^{\mathrm{a}}$ & $3.361^{\mathrm{a}}$ & $3.160^{\mathrm{a}}$ & 52.5 & NS \\
\hline $\mathrm{N} / \mathrm{Fe}$ & $0.188^{\mathrm{a}}$ & $0.258^{\mathrm{a}}$ & $0.240^{\mathrm{a}}$ & $0.241^{\mathrm{a}}$ & $0.225^{\mathrm{a}}$ & $0.245^{\mathrm{a}}$ & $0.256^{\mathrm{a}}$ & 38.2 & NS \\
\hline $\mathrm{N} / \mathrm{Mn}$ & $0.229^{\mathrm{a}}$ & $0.245^{\mathrm{a}}$ & $0.245^{\mathrm{a}}$ & $0.250^{\mathrm{a}}$ & $0.233^{\mathrm{a}}$ & $0.277^{\mathrm{a}}$ & $0.214^{\mathrm{a}}$ & 57.0 & NS \\
\hline $\bar{N} / \mathrm{Zn}$ & $4.607^{\mathrm{a}}$ & $4.156^{\mathrm{a}}$ & $3.888^{\mathrm{b}}$ & $4.732^{\mathrm{a}}$ & $4.196^{\mathrm{a}}$ & $3.555^{\mathrm{b}}$ & $4.335^{\mathrm{a}}$ & 21.2 & $* *$ \\
\hline $\mathrm{P} / \mathrm{N}$ & $0.045^{\mathrm{b}}$ & $0.046^{\mathrm{b}}$ & $0.050^{\mathrm{a}}$ & $0.048^{\mathrm{a}}$ & $0.046^{\mathrm{b}}$ & $0.044^{\mathrm{b}}$ & $0.050^{\mathrm{a}}$ & 14.4 & $*$ \\
\hline $\mathrm{P} / \mathrm{K}$ & $0.096^{\mathrm{a}}$ & $0.099^{\mathrm{a}}$ & $0.103^{\mathrm{a}}$ & $0.111^{\mathrm{a}}$ & $0.097^{\mathrm{a}}$ & $0.107^{\mathrm{a}}$ & $0.112^{\mathrm{a}}$ & 29.1 & NS \\
\hline $\mathrm{P} / \mathrm{Ca}$ & $0.055^{\mathrm{c}}$ & $0.074^{\mathrm{a}}$ & $0.064^{b}$ & $0.064^{b}$ & $0.047^{\mathrm{c}}$ & $0.061^{\mathrm{b}}$ & $0.073^{\mathrm{a}}$ & 27.1 & $* *$ \\
\hline $\mathrm{P} / \mathrm{Mg}$ & $0.344^{\mathrm{a}}$ & $0.384^{\mathrm{a}}$ & $0.437^{\mathrm{a}}$ & $0.366^{\mathrm{a}}$ & $0.337^{\mathrm{a}}$ & $0.342^{\mathrm{a}}$ & $0.367^{\mathrm{a}}$ & 35.5 & NS \\
\hline $\mathrm{P} / \mathrm{S}$ & $1.077^{\mathrm{a}}$ & $0.967^{\mathrm{a}}$ & $0.882^{b}$ & $0.944^{\mathrm{a}}$ & $0.956^{\mathrm{a}}$ & $0.738^{\mathrm{c}}$ & $1.033^{\mathrm{a}}$ & 23.7 & $* *$ \\
\hline $\mathrm{P} / \mathrm{B}$ & $0.016^{\mathrm{a}}$ & $0.022^{\mathrm{a}}$ & $0.020^{\mathrm{a}}$ & $0.019^{\mathrm{a}}$ & $0.016^{\mathrm{a}}$ & $0.017^{\mathrm{a}}$ & $0.019^{\mathrm{a}}$ & 46.2 & NS \\
\hline $\mathrm{P} / \mathrm{Cu}$ & $0.164^{\mathrm{a}}$ & $0.197^{\mathrm{a}}$ & $0.160^{\mathrm{a}}$ & $0.167^{\mathrm{a}}$ & $0.193^{\mathrm{a}}$ & $0.152^{\mathrm{a}}$ & $0.163^{\mathrm{a}}$ & 56.0 & NS \\
\hline $\mathrm{P} / \mathrm{Fe}$ & $0.008^{\mathrm{a}}$ & $0.012^{\mathrm{a}}$ & $0.012^{\mathrm{a}}$ & $0.011^{\mathrm{a}}$ & $0.010^{\mathrm{a}}$ & $0.011^{\mathrm{a}}$ & $0.013^{\mathrm{a}}$ & 38.0 & NS \\
\hline $\mathrm{P} / \mathrm{Mn}$ & $0.010^{\mathrm{a}}$ & $0.012^{\mathrm{a}}$ & $0.012^{\mathrm{a}}$ & $0.012^{\mathrm{a}}$ & $0.011^{\mathrm{a}}$ & $0.012^{\mathrm{a}}$ & $0.011^{\mathrm{a}}$ & 61.5 & NS \\
\hline $\mathrm{P} / \mathrm{Zn}$ & $0.207^{\mathrm{a}}$ & $0.191^{\mathrm{a}}$ & $0.196^{\mathrm{a}}$ & $0.227^{\mathrm{a}}$ & $0.195^{\mathrm{b}}$ & $0.157^{b}$ & $0.220^{\mathrm{a}}$ & 24.9 & $* *$ \\
\hline $\mathrm{K} / \mathrm{N}$ & $0.485^{\mathrm{a}}$ & $0.490^{\mathrm{a}}$ & $0.524^{\mathrm{a}}$ & $0.458^{\mathrm{a}}$ & $0.506^{\mathrm{a}}$ & $0.440^{\mathrm{a}}$ & $0.489^{\mathrm{a}}$ & 22.6 & NS \\
\hline $\mathrm{K} / \mathrm{P}$ & $10.98^{\mathrm{a}}$ & $10.94^{\mathrm{a}}$ & $10.71^{\mathrm{a}}$ & $9.839^{\mathrm{a}}$ & $11.36^{\mathrm{a}}$ & $10.22^{\mathrm{a}}$ & $10.08^{\mathrm{a}}$ & 33.7 & NS \\
\hline $\mathrm{K} / \mathrm{Ca}$ & $0.611^{\mathrm{a}}$ & $0.820^{\mathrm{a}}$ & $0.683^{\mathrm{a}}$ & $0.623^{\mathrm{a}}$ & $0.528^{\mathrm{a}}$ & $0.631^{\mathrm{a}}$ & $0.743^{\mathrm{a}}$ & 44.0 & NS \\
\hline $\mathrm{K} / \mathrm{Mg}$ & $3.787^{\mathrm{a}}$ & $4.228^{\mathrm{a}}$ & $4.650^{\mathrm{a}}$ & $3.526^{\mathrm{a}}$ & $3.874^{\mathrm{a}}$ & $3.556^{\mathrm{a}}$ & $3.667^{\mathrm{a}}$ & 46.3 & NS \\
\hline $\mathrm{K} / \mathrm{S}$ & $11.53^{\mathrm{a}}$ & $10.22^{\mathrm{a}}$ & $8.895^{\mathrm{b}}$ & $8.856^{\mathrm{b}}$ & $10.36^{\mathrm{a}}$ & $7.393^{b}$ & $9.895^{\mathrm{a}}$ & 27.2 & $* *$ \\
\hline $\mathrm{K} / \mathrm{B}$ & $0.175^{\mathrm{a}}$ & $0.240^{\mathrm{a}}$ & $0.205^{\mathrm{a}}$ & $0.174^{\mathrm{a}}$ & $0.183^{\mathrm{a}}$ & $0.166^{\mathrm{a}}$ & $0.188^{\mathrm{a}}$ & 47.1 & NS \\
\hline $\mathrm{K} / \mathrm{Cu}$ & $1.764^{\mathrm{a}}$ & $2.022^{\mathrm{a}}$ & $1.531^{\mathrm{a}}$ & $1.553^{\mathrm{a}}$ & $2.049^{\mathrm{a}}$ & $1.440^{\mathrm{a}}$ & $1.479^{\mathrm{a}}$ & 52.0 & NS \\
\hline $\mathrm{K} / \mathrm{Fe}$ & $0.091^{\mathrm{a}}$ & $0.127^{\mathrm{a}}$ & $0.123^{\mathrm{a}}$ & $0.109^{\mathrm{a}}$ & $0.115^{\mathrm{a}}$ & $0.107^{\mathrm{a}}$ & $0.126^{\mathrm{a}}$ & 45.5 & NS \\
\hline $\mathrm{K} / \mathrm{Mn}$ & $0.112^{\mathrm{a}}$ & $0.117^{\mathrm{a}}$ & $0.127^{\mathrm{a}}$ & $0.119^{\mathrm{a}}$ & $0.121^{\mathrm{a}}$ & $0.125^{\mathrm{a}}$ & $0.109^{\mathrm{a}}$ & 61.5 & NS \\
\hline$\overline{\mathrm{K} / \mathrm{Zn}}$ & $2.223^{\mathrm{a}}$ & $2.080^{\mathrm{a}}$ & $2.008^{\mathrm{a}}$ & $2.149^{\mathrm{a}}$ & $2.118^{\mathrm{a}}$ & $1.570^{\mathrm{b}}$ & $2.126^{\mathrm{a}}$ & 30.6 & NS \\
\hline $\mathrm{Ca} / \mathrm{N}$ & $0.861^{b}$ & $0.661^{\mathrm{c}}$ & $0.813^{b}$ & $0.798^{\mathrm{b}}$ & $1.046^{\mathrm{a}}$ & $0.758^{\mathrm{c}}$ & $0.735^{\mathrm{c}}$ & 23.9 & $* *$ \\
\hline $\mathrm{Ca} / \mathrm{P}$ & $19.39^{b}$ & $14.43^{\mathrm{c}}$ & $16.33^{\mathrm{c}}$ & $16.78^{\mathrm{c}}$ & $22.78^{\mathrm{a}}$ & $17.13^{\mathrm{c}}$ & $14.79^{c}$ & 25.0 & $* *$ \\
\hline $\mathrm{Ca} / \mathrm{K}$ & $1.866^{\mathrm{a}}$ & $1.450^{\mathrm{a}}$ & $1.671^{\mathrm{a}}$ & $1.866^{\mathrm{a}}$ & $2.202^{\mathrm{a}}$ & $1.896^{\mathrm{a}}$ & $1.662^{\mathrm{a}}$ & 41.6 & NS \\
\hline $\mathrm{Ca} / \mathrm{Mg}$ & $6.315^{\mathrm{a}}$ & $5.207^{b}$ & $6.783^{\mathrm{a}}$ & $5.686^{\mathrm{b}}$ & $7.143^{\mathrm{a}}$ & $5.621^{\mathrm{b}}$ & $5.059^{b}$ & 23.7 & $* *$ \\
\hline $\mathrm{Ca} / \mathrm{S}$ & $20.70^{\mathrm{a}}$ & $13.46^{\mathrm{b}}$ & $13.99^{b}$ & $15.30^{\mathrm{b}}$ & $21.49^{\mathrm{a}}$ & $12.48^{\mathrm{b}}$ & $14.89^{b}$ & 28.6 & $* *$ \\
\hline $\mathrm{Ca} / \mathrm{B}$ & $0.312^{\mathrm{a}}$ & $0.304^{\mathrm{a}}$ & $0.304^{\mathrm{a}}$ & $0.287^{\mathrm{a}}$ & $0.370^{\mathrm{a}}$ & $0.281^{\mathrm{a}}$ & $0.273^{\mathrm{a}}$ & 42.9 & NS \\
\hline$\overline{\mathrm{Ca} / \mathrm{Cu}}$ & $3.108^{b}$ & $2.769^{b}$ & $2.607^{b}$ & $2.767^{b}$ & $4.268^{\mathrm{a}}$ & $2.673^{b}$ & $2.390^{\mathrm{b}}$ & 59.2 & $*$ \\
\hline $\mathrm{Ca} / \mathrm{Fe}$ & $0.164^{\mathrm{a}}$ & $0.163^{\mathrm{a}}$ & $0.188^{\mathrm{a}}$ & $0.186^{\mathrm{a}}$ & $0.230^{\mathrm{a}}$ & $0.180^{\mathrm{a}}$ & $0.187^{\mathrm{a}}$ & 37.7 & NS \\
\hline $\mathrm{Ca} / \mathrm{Mn}$ & $0.198^{\mathrm{a}}$ & $0.160^{\mathrm{a}}$ & $0.196^{\mathrm{a}}$ & $0.192^{\mathrm{a}}$ & $0.223^{\mathrm{a}}$ & $0.207^{\mathrm{a}}$ & $0.155^{\mathrm{a}}$ & 57.2 & NS \\
\hline $\mathrm{Ca} / \mathrm{Zn}$ & $3.906^{\mathrm{a}}$ & $2.656^{\mathrm{b}}$ & $3.121^{b}$ & $3.743^{\mathrm{a}}$ & $4.346^{\mathrm{a}}$ & $2.629^{\mathrm{b}}$ & $3.169^{b}$ & 28.0 & $* *$ \\
\hline $\mathrm{Mg} / \mathrm{N}$ & $0.139^{\mathrm{a}}$ & $0.130^{\mathrm{a}}$ & $0.126^{\mathrm{a}}$ & $0.144^{\mathrm{a}}$ & $0.158^{\mathrm{a}}$ & $0.140^{\mathrm{a}}$ & $0.147^{\mathrm{a}}$ & 30.2 & NS \\
\hline $\mathrm{Mg} / \mathrm{P}$ & $3.161^{\mathrm{a}}$ & $2.851^{\mathrm{a}}$ & $2.529^{\mathrm{a}}$ & $3.044^{\mathrm{a}}$ & $3.434^{\mathrm{a}}$ & $3.188^{\mathrm{a}}$ & $2.966^{\mathrm{a}}$ & 31.2 & NS \\
\hline$\overline{\mathrm{Mg} / \mathrm{K}}$ & $0.305^{\mathrm{a}}$ & $0.287^{\mathrm{a}}$ & $0.263^{\mathrm{a}}$ & $0.340^{\mathrm{a}}$ & $0.340^{\mathrm{a}}$ & $0.356^{\mathrm{a}}$ & $0.329^{\mathrm{a}}$ & 50.2 & NS \\
\hline $\mathrm{Mg} / \mathrm{Ca}$ & $0.164^{b}$ & $0.198^{\mathrm{a}}$ & $0.155^{\mathrm{b}}$ & $0.182^{\mathrm{a}}$ & $0.150^{b}$ & $0.186^{\mathrm{a}}$ & $0.202^{\mathrm{a}}$ & 19.7 & $* *$ \\
\hline $\mathrm{Mg} / \mathrm{S}$ & $3.334^{\mathrm{a}}$ & $2.635^{\mathrm{b}}$ & $2.169^{b}$ & $2.762^{b}$ & $3.255^{\mathrm{a}}$ & $2.297^{b}$ & $2.977^{\mathrm{a}}$ & 32.9 & $* *$ \\
\hline $\mathrm{Mg} / \mathrm{B}$ & $0.049^{\mathrm{a}}$ & $0.058^{\mathrm{a}}$ & $0.046^{\mathrm{a}}$ & $0.051^{\mathrm{a}}$ & $0.055^{\mathrm{a}}$ & $0.050^{\mathrm{a}}$ & $0.054^{\mathrm{a}}$ & 39.2 & NS \\
\hline $\mathrm{Mg} / \mathrm{Cu}$ & $0.489^{\mathrm{a}}$ & $0.553^{\mathrm{a}}$ & $0.412^{\mathrm{a}}$ & $0.487^{\mathrm{a}}$ & $0.636^{\mathrm{a}}$ & $0.487^{\mathrm{a}}$ & $0.465^{\mathrm{a}}$ & 62.8 & NS \\
\hline$\overline{\mathrm{Mg} / \mathrm{Fe}}$ & $0.026^{\mathrm{a}}$ & $0.032^{\mathrm{a}}$ & $0.029^{\mathrm{a}}$ & $0.034^{\mathrm{a}}$ & $0.035^{\mathrm{a}}$ & $0.033^{\mathrm{a}}$ & $0.037^{\mathrm{a}}$ & 41.2 & NS \\
\hline $\mathrm{Mg} / \mathrm{Mn}$ & $0.031^{\mathrm{a}}$ & $0.031^{\mathrm{a}}$ & $0.030^{\mathrm{a}}$ & $0.034^{\mathrm{a}}$ & $0.032^{\mathrm{a}}$ & $0.037^{\mathrm{a}}$ & $0.031^{\mathrm{a}}$ & 58.2 & NS \\
\hline $\mathrm{Mg} / \mathrm{Zn}$ & $0.640^{\mathrm{a}}$ & $0.521^{b}$ & $0.479^{b}$ & $0.674^{\mathrm{a}}$ & $0.653^{\mathrm{a}}$ & $0.485^{b}$ & $0.633^{\mathrm{a}}$ & 32.1 & $* *$ \\
\hline $\mathrm{S} / \mathrm{N}$ & $0.043^{b}$ & $0.049^{b}$ & $0.061^{\mathrm{a}}$ & $0.053^{b}$ & $0.049^{b}$ & $0.062^{\mathrm{a}}$ & $0.050^{\mathrm{b}}$ & 21.9 & $* *$ \\
\hline $\mathrm{S} / \mathrm{P}$ & $0.981^{b}$ & $1.098^{\mathrm{b}}$ & $1.230^{\mathrm{a}}$ & $1.130^{\mathrm{b}}$ & $1.092^{\mathrm{b}}$ & $1.405^{\mathrm{a}}$ & $1.021^{\mathrm{b}}$ & 25.8 & $* *$ \\
\hline $\mathrm{S} / \mathrm{K}$ & $0.092^{\mathrm{b}}$ & $0.106^{b}$ & $0.119^{b}$ & $0.120^{\mathrm{b}}$ & $0.101^{\mathrm{b}}$ & $0.148^{\mathrm{a}}$ & $0.109^{b}$ & 27.8 & $* *$ \\
\hline $\mathrm{S} / \mathrm{Ca}$ & $0.053^{b}$ & $0.078^{\mathrm{a}}$ & $0.077^{\mathrm{a}}$ & $0.069^{\mathrm{a}}$ & $0.050^{\mathrm{b}}$ & $0.084^{\mathrm{a}}$ & $0.073^{\mathrm{a}}$ & 26.7 & $* *$ \\
\hline $\mathrm{S} / \mathrm{Mg}$ & $0.328^{\mathrm{b}}$ & $0.401^{\mathrm{b}}$ & $0.524^{\mathrm{a}}$ & $0.391^{\mathrm{b}}$ & $0.364^{b}$ & $0.469^{\mathrm{a}}$ & $0.363^{b}$ & 34.7 & $* *$ \\
\hline $\mathrm{S} / \mathrm{B}$ & $0.015^{\mathrm{b}}$ & $0.023^{\mathrm{a}}$ & $0.023^{\mathrm{a}}$ & $0.019^{b}$ & $0.018^{\mathrm{b}}$ & $0.022^{\mathrm{a}}$ & $0.019^{\mathrm{b}}$ & 34.9 & $* *$ \\
\hline $\mathrm{S} / \mathrm{Cu}$ & $0.158^{\mathrm{a}}$ & $0.207^{\mathrm{a}}$ & $0.181^{\mathrm{a}}$ & $0.181^{\mathrm{a}}$ & $0.205^{\mathrm{a}}$ & $0.210^{\mathrm{a}}$ & $0.159^{\mathrm{a}}$ & 55.6 & NS \\
\hline $\mathrm{S} / \mathrm{Fe}$ & $0.008^{\mathrm{b}}$ & $0.013^{\mathrm{a}}$ & $0.014^{\mathrm{a}}$ & $0.012^{\mathrm{a}}$ & $0.011^{\mathrm{b}}$ & $0.015^{\mathrm{a}}$ & $0.013^{\mathrm{a}}$ & 39.2 & $* *$ \\
\hline
\end{tabular}




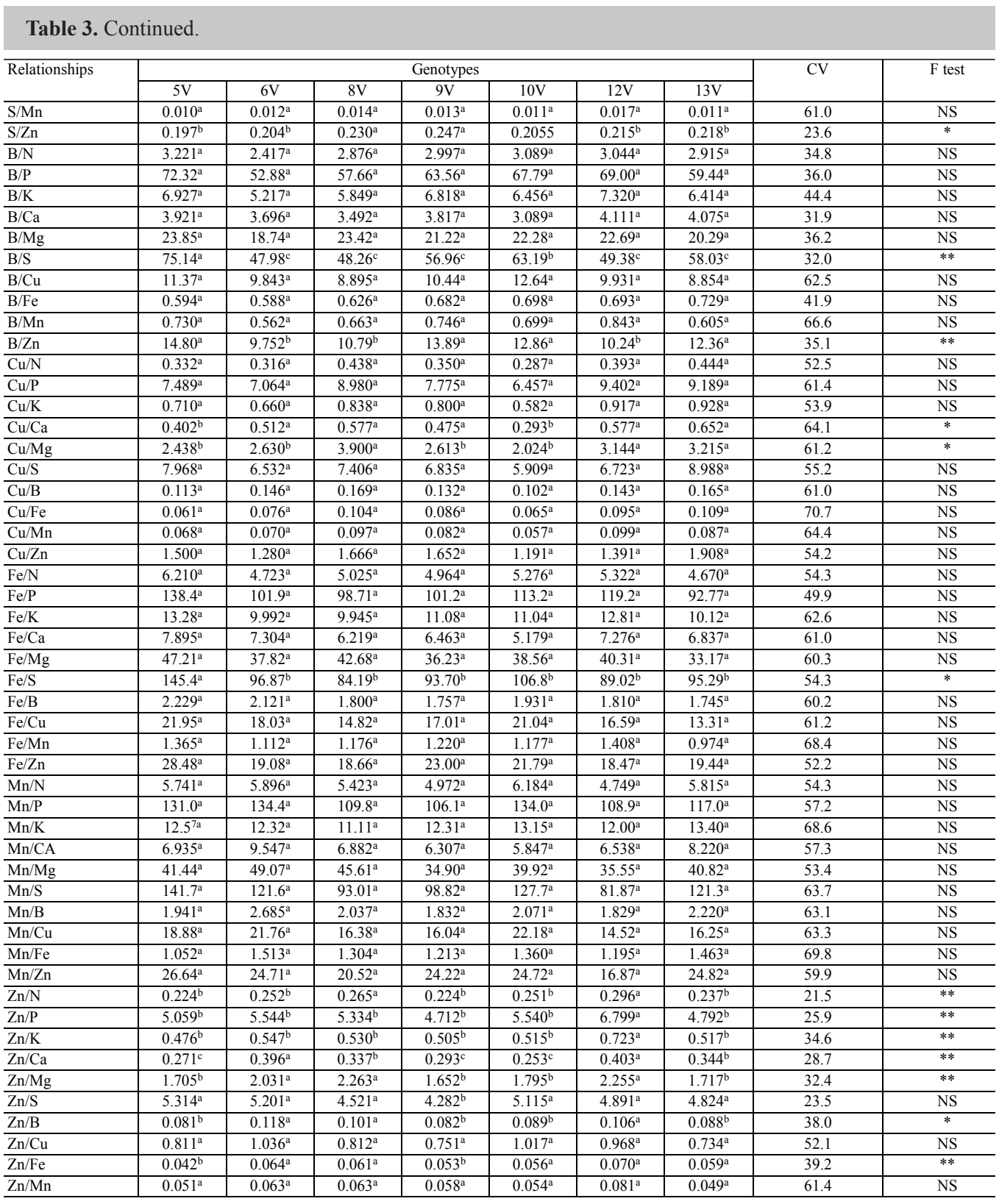

For each nutrient relationship, the mean values followed by different letters indicate significant differences between genotypes according to the Scott-Knott test at $5 \%$ probability. $\mathrm{NS}=$ not significant; **highly significant $(\mathrm{P} \leq 0.01)$; *significant $(\mathrm{P} \leq 0.05)$.

Similar results were observed in the leaves of conilon coffee seedlings by Covre et al. (2013). Differences in the nutritional status of the leaf may be associated with different nutritional requirements over the course of a year (Partelli et al., 2014b; Marré et al., 2015).

Genetics and Molecular Research 15 (4): gmr.15048839 
Table 4. Mean values, CV, and F- and Scott-Knott test results for foliar nutrient relationships in seven conilon genotypes of the clonal variety Vitória Incaper 8142 with crop yield either equal to or greater than 100 bags per hectare (average from 2012 and 2013 harvests) during the bean-filling stage.

\begin{tabular}{|c|c|c|c|c|c|c|c|c|c|}
\hline \multirow[t]{2}{*}{ Relationships } & \multicolumn{7}{|c|}{ Genotypes } & \multirow[t]{2}{*}{$\mathrm{CV}$} & \multirow[t]{2}{*}{ F test } \\
\hline & $5 \mathrm{~V}$ & $6 \mathrm{~V}$ & $8 \mathrm{~V}$ & $9 \mathrm{~V}$ & $10 \mathrm{~V}$ & $12 \mathrm{~V}$ & $13 \mathrm{~V}$ & & \\
\hline $\mathrm{N} / \mathrm{P}$ & $22.62^{\mathrm{a}}$ & $22.13^{\mathrm{a}}$ & $20.21^{\mathrm{a}}$ & $21.30^{\mathrm{a}}$ & $22.00^{\mathrm{a}}$ & $23.10^{\mathrm{a}}$ & $20.21^{\mathrm{a}}$ & 17.0 & NS \\
\hline $\mathrm{N} / \mathrm{K}$ & $2.132^{\mathrm{a}}$ & $2.138^{\mathrm{a}}$ & $2.022^{\mathrm{a}}$ & $2.289^{\mathrm{a}}$ & $2.062^{\mathrm{a}}$ & $2.404^{\mathrm{a}}$ & $2.176^{\mathrm{a}}$ & 23.9 & NS \\
\hline $\mathrm{N} / \mathrm{Ca}$ & $1.231^{\mathrm{b}}$ & $1.619^{\mathrm{a}}$ & $1.283^{\mathrm{b}}$ & $1.332^{\mathrm{b}}$ & $1.016^{\mathrm{c}}$ & $1.396^{\mathrm{a}}$ & $1.460^{\mathrm{a}}$ & 26.9 & $* *$ \\
\hline $\mathrm{N} / \mathrm{Mg}$ & $7.618^{\mathrm{a}}$ & $8.333^{\mathrm{a}}$ & $8.706^{\mathrm{a}}$ & $7.519^{\mathrm{a}}$ & $7.341^{\mathrm{a}}$ & $7.808^{\mathrm{a}}$ & $7.293^{\mathrm{a}}$ & 32.2 & NS \\
\hline $\mathrm{N} / \mathrm{S}$ & $23.97^{\mathrm{a}}$ & $20.81^{b}$ & $17.52^{\mathrm{c}}$ & $19.56^{\mathrm{b}}$ & $20.58^{b}$ & $16.87^{\mathrm{c}}$ & $20.46^{b}$ & 19.7 & $* *$ \\
\hline $\bar{N} / \mathrm{B}$ & $0.362^{\mathrm{a}}$ & $0.484^{\mathrm{a}}$ & $0.395^{\mathrm{a}}$ & $0.379^{\mathrm{a}}$ & $0.357^{\mathrm{a}}$ & $0.382^{\mathrm{a}}$ & $0.381^{\mathrm{a}}$ & 42.4 & NS \\
\hline $\mathrm{N} / \mathrm{Cu}$ & $3.660^{\mathrm{a}}$ & $4.229^{\mathrm{a}}$ & $3.141^{\mathrm{a}}$ & $3.382^{\mathrm{a}}$ & $4.119^{\mathrm{a}}$ & $3.361^{\mathrm{a}}$ & $3.160^{\mathrm{a}}$ & 52.5 & NS \\
\hline $\mathrm{N} / \mathrm{Fe}$ & $0.188^{\mathrm{a}}$ & $0.258^{\mathrm{a}}$ & $0.240^{\mathrm{a}}$ & $0.241^{\mathrm{a}}$ & $0.225^{\mathrm{a}}$ & $0.245^{\mathrm{a}}$ & $0.256^{\mathrm{a}}$ & 38.2 & NS \\
\hline $\mathrm{N} / \mathrm{Mn}$ & $0.229^{\mathrm{a}}$ & $0.245^{\mathrm{a}}$ & $0.245^{\mathrm{a}}$ & $0.250^{\mathrm{a}}$ & $0.233^{\mathrm{a}}$ & $0.277^{\mathrm{a}}$ & $0.214^{\mathrm{a}}$ & 57.0 & NS \\
\hline $\mathrm{N} / \mathrm{Zn}$ & $4.607^{\mathrm{a}}$ & $4.156^{\mathrm{a}}$ & $3.888^{\mathrm{b}}$ & $4.732^{\mathrm{a}}$ & $4.196^{\mathrm{a}}$ & $3.555^{\mathrm{b}}$ & $4.335^{\mathrm{a}}$ & 21.2 & $* *$ \\
\hline $\mathrm{P} / \mathrm{N}$ & $0.045^{\mathrm{b}}$ & $0.046^{\mathrm{b}}$ & $0.050^{\mathrm{a}}$ & $0.048^{\mathrm{a}}$ & $0.046^{\mathrm{b}}$ & $0.044^{b}$ & $0.050^{\mathrm{a}}$ & 14.4 & $*$ \\
\hline $\mathrm{P} / \mathrm{K}$ & $0.096^{\mathrm{a}}$ & $0.099^{\mathrm{a}}$ & $0.103^{\mathrm{a}}$ & $0.111^{\mathrm{a}}$ & $0.097^{\mathrm{a}}$ & $0.107^{\mathrm{a}}$ & $0.112^{\mathrm{a}}$ & 29.1 & NS \\
\hline $\mathrm{P} / \mathrm{Ca}$ & $0.055^{\mathrm{c}}$ & $0.074^{\mathrm{a}}$ & $0.064^{\mathrm{b}}$ & $0.064^{\mathrm{b}}$ & $0.047^{\mathrm{c}}$ & $0.061^{\mathrm{b}}$ & $0.073^{\mathrm{a}}$ & 27.1 & $* *$ \\
\hline $\mathrm{P} / \mathrm{Mg}$ & $0.344^{\mathrm{a}}$ & $0.384^{\mathrm{a}}$ & $0.437^{\mathrm{a}}$ & $0.366^{\mathrm{a}}$ & $0.337^{\mathrm{a}}$ & $0.342^{\mathrm{a}}$ & $0.367^{\mathrm{a}}$ & 35.5 & NS \\
\hline $\mathrm{P} / \mathrm{S}$ & $1.077^{\mathrm{a}}$ & $0.967^{\mathrm{a}}$ & $0.882^{b}$ & $0.944^{\mathrm{a}}$ & $0.956^{\mathrm{a}}$ & $0.738^{\mathrm{c}}$ & $1.033^{\mathrm{a}}$ & 23.7 & $* *$ \\
\hline $\mathrm{P} / \mathrm{B}$ & $0.016^{\mathrm{a}}$ & $0.022^{\mathrm{a}}$ & $0.020^{\mathrm{a}}$ & $0.019^{\mathrm{a}}$ & $0.016^{\mathrm{a}}$ & $0.017^{\mathrm{a}}$ & $0.019^{\mathrm{a}}$ & 46.2 & NS \\
\hline $\mathrm{P} / \mathrm{Cu}$ & $0.164^{\mathrm{a}}$ & $0.197^{\mathrm{a}}$ & $0.160^{\mathrm{a}}$ & $0.167^{\mathrm{a}}$ & $0.193^{\mathrm{a}}$ & $0.152^{\mathrm{a}}$ & $0.163^{\mathrm{a}}$ & 56.0 & NS \\
\hline $\mathrm{P} / \mathrm{Fe}$ & $0.008^{\mathrm{a}}$ & $0.012^{\mathrm{a}}$ & $0.012^{\mathrm{a}}$ & $0.011^{\mathrm{a}}$ & $0.010^{\mathrm{a}}$ & $0.011^{\mathrm{a}}$ & $0.013^{\mathrm{a}}$ & 38.0 & NS \\
\hline $\mathrm{P} / \mathrm{Mn}$ & $0.010^{\mathrm{a}}$ & $0.012^{\mathrm{a}}$ & $0.012^{\mathrm{a}}$ & $0.012^{\mathrm{a}}$ & $0.011^{\mathrm{a}}$ & $0.012^{\mathrm{a}}$ & $0.011^{\mathrm{a}}$ & 61.5 & NS \\
\hline $\mathrm{P} / \mathrm{Zn}$ & $0.207^{\mathrm{a}}$ & $0.191^{\mathrm{a}}$ & $0.196^{\mathrm{a}}$ & $0.227^{\mathrm{a}}$ & $0.195^{\mathrm{b}}$ & $0.157^{b}$ & $0.220^{\mathrm{a}}$ & 24.9 & $* *$ \\
\hline $\mathrm{K} / \mathrm{N}$ & $0.485^{\mathrm{a}}$ & $0.490^{\mathrm{a}}$ & $0.524^{\mathrm{a}}$ & $0.458^{\mathrm{a}}$ & $0.506^{\mathrm{a}}$ & $0.440^{\mathrm{a}}$ & $0.489^{\mathrm{a}}$ & 22.6 & NS \\
\hline $\mathrm{K} / \mathrm{P}$ & $10.98^{\mathrm{a}}$ & $10.94^{\mathrm{a}}$ & $10.71^{\mathrm{a}}$ & $9.839^{\mathrm{a}}$ & $11.36^{\mathrm{a}}$ & $10.22^{\mathrm{a}}$ & $10.08^{\mathrm{a}}$ & 33.7 & NS \\
\hline $\mathrm{K} / \mathrm{Ca}$ & $0.611^{\mathrm{a}}$ & $0.820^{\mathrm{a}}$ & $0.683^{\mathrm{a}}$ & $0.623^{\mathrm{a}}$ & $0.528^{\mathrm{a}}$ & $0.631^{\mathrm{a}}$ & $0.743^{\mathrm{a}}$ & 44.0 & NS \\
\hline $\mathrm{K} / \mathrm{Mg}$ & $3.787^{\mathrm{a}}$ & $4.228^{\mathrm{a}}$ & $4.650^{\mathrm{a}}$ & $3.526^{\mathrm{a}}$ & $3.874^{\mathrm{a}}$ & $3.556^{\mathrm{a}}$ & $3.667^{\mathrm{a}}$ & 46.3 & NS \\
\hline $\mathrm{K} / \mathrm{S}$ & $11.53^{\mathrm{a}}$ & $10.22^{\mathrm{a}}$ & $8.895^{b}$ & $8.856^{\mathrm{b}}$ & $10.36^{\mathrm{a}}$ & $7.393^{b}$ & $9.895^{\mathrm{a}}$ & 27.2 & ** \\
\hline $\mathrm{K} / \mathrm{B}$ & $0.175^{\mathrm{a}}$ & $0.240^{\mathrm{a}}$ & $0.205^{\mathrm{a}}$ & $0.174^{\mathrm{a}}$ & $0.183^{\mathrm{a}}$ & $0.166^{\mathrm{a}}$ & $0.188^{\mathrm{a}}$ & 47.1 & NS \\
\hline $\mathrm{K} / \mathrm{Cu}$ & $1.764^{\mathrm{a}}$ & $2.022^{\mathrm{a}}$ & $1.531^{\mathrm{a}}$ & $1.553^{\mathrm{a}}$ & $2.049^{\mathrm{a}}$ & $1.440^{\mathrm{a}}$ & $1.479^{\mathrm{a}}$ & 52.0 & NS \\
\hline $\mathrm{K} / \mathrm{Fe}$ & $0.091^{\mathrm{a}}$ & $0.127^{\mathrm{a}}$ & $0.123^{\mathrm{a}}$ & $0.109^{\mathrm{a}}$ & $0.115^{\mathrm{a}}$ & $0.107^{\mathrm{a}}$ & $0.126^{\mathrm{a}}$ & 45.5 & NS \\
\hline $\mathrm{K} / \mathrm{Mn}$ & $0.112^{\mathrm{a}}$ & $0.117^{\mathrm{a}}$ & $0.127^{\mathrm{a}}$ & $0.119^{\mathrm{a}}$ & $0.121^{\mathrm{a}}$ & $0.125^{\mathrm{a}}$ & $0.109^{\mathrm{a}}$ & 61.5 & NS \\
\hline $\mathrm{K} / \mathrm{Zn}$ & $2.223^{\mathrm{a}}$ & $2.080^{\mathrm{a}}$ & $2.008^{\mathrm{a}}$ & $2.149^{\mathrm{a}}$ & $2.118^{\mathrm{a}}$ & $1.570^{\mathrm{b}}$ & $2.126^{\mathrm{a}}$ & 30.6 & NS \\
\hline $\mathrm{Ca} / \mathrm{N}$ & $0.861^{\mathrm{b}}$ & $0.661^{\mathrm{c}}$ & $0.813^{\mathrm{b}}$ & $0.798^{\mathrm{b}}$ & $1.046^{\mathrm{a}}$ & $0.758^{\mathrm{c}}$ & $0.735^{\mathrm{c}}$ & 23.9 & $* *$ \\
\hline $\mathrm{Ca} / \mathrm{P}$ & $19.39^{\mathrm{b}}$ & $14.43^{\mathrm{c}}$ & $16.33^{\mathrm{c}}$ & $16.78^{\mathrm{c}}$ & $22.78^{\mathrm{a}}$ & $17.13^{\mathrm{c}}$ & $14.79^{\mathrm{c}}$ & 25.0 & ** \\
\hline $\mathrm{Ca} / \mathrm{K}$ & $1.866^{\mathrm{a}}$ & $1.450^{\mathrm{a}}$ & $1.671^{\mathrm{a}}$ & $1.866^{\mathrm{a}}$ & $2.202^{\mathrm{a}}$ & $1.896^{\mathrm{a}}$ & $1.662^{\mathrm{a}}$ & 41.6 & NS \\
\hline $\mathrm{Ca} / \mathrm{Mg}$ & $6.315^{\mathrm{a}}$ & $5.207^{b}$ & $6.783^{\mathrm{a}}$ & $5.686^{b}$ & $7.143^{\mathrm{a}}$ & $5.621^{b}$ & $5.059^{b}$ & 23.7 & ** \\
\hline $\mathrm{Ca} / \mathrm{S}$ & $20.70^{\mathrm{a}}$ & $13.46^{\mathrm{b}}$ & $13.99^{\mathrm{b}}$ & $15.30^{\mathrm{b}}$ & $21.49^{\mathrm{a}}$ & $12.48^{b}$ & $14.89^{b}$ & 28.6 & ** \\
\hline $\mathrm{Ca} / \mathrm{B}$ & $0.312^{\mathrm{a}}$ & $0.304^{\mathrm{a}}$ & $0.304^{\mathrm{a}}$ & $0.287^{\mathrm{a}}$ & $0.370^{\mathrm{a}}$ & $0.281^{\mathrm{a}}$ & $0.273^{\mathrm{a}}$ & 42.9 & NS \\
\hline $\mathrm{Ca} / \mathrm{Cu}$ & $3.108^{b}$ & $2.769^{b}$ & $2.607^{b}$ & $2.767^{b}$ & $4.268^{\mathrm{a}}$ & $2.673^{\mathrm{b}}$ & $2.390^{\mathrm{b}}$ & 59.2 & $*$ \\
\hline $\mathrm{Ca} / \mathrm{Fe}$ & $0.164^{\mathrm{a}}$ & $0.163^{\mathrm{a}}$ & $0.188^{\mathrm{a}}$ & $0.186^{\mathrm{a}}$ & $0.230^{\mathrm{a}}$ & $0.180^{\mathrm{a}}$ & $0.187^{\mathrm{a}}$ & 37.7 & NS \\
\hline $\mathrm{Ca} / \mathrm{Mn}$ & $0.198^{\mathrm{a}}$ & $0.160^{\mathrm{a}}$ & $0.196^{\mathrm{a}}$ & $0.192^{\mathrm{a}}$ & $0.223^{\mathrm{a}}$ & $0.207^{\mathrm{a}}$ & $0.155^{\mathrm{a}}$ & 57.2 & NS \\
\hline $\mathrm{Ca} / \mathrm{Zn}$ & $3.906^{\mathrm{a}}$ & $2.656^{\mathrm{b}}$ & $3.121^{b}$ & $3.743^{\mathrm{a}}$ & $4.346^{\mathrm{a}}$ & $2.629^{b}$ & $3.169^{b}$ & 28.0 & $* *$ \\
\hline $\mathrm{Mg} / \mathrm{N}$ & $0.139^{\mathrm{a}}$ & $0.130^{\mathrm{a}}$ & $0.126^{\mathrm{a}}$ & $0.144^{\mathrm{a}}$ & $0.158^{\mathrm{a}}$ & $0.140^{\mathrm{a}}$ & $0.147^{\mathrm{a}}$ & 30.2 & NS \\
\hline $\mathrm{Mg} / \mathrm{P}$ & $3.161^{\mathrm{a}}$ & $2.851^{\mathrm{a}}$ & $2.529^{\mathrm{a}}$ & $3.044^{\mathrm{a}}$ & $3.434^{\mathrm{a}}$ & $3.188^{\mathrm{a}}$ & $2.966^{\mathrm{a}}$ & 31.2 & NS \\
\hline $\mathrm{Mg} / \mathrm{K}$ & $0.305^{\mathrm{a}}$ & $0.287^{\mathrm{a}}$ & $0.263^{\mathrm{a}}$ & $0.340^{\mathrm{a}}$ & $0.340^{\mathrm{a}}$ & $0.356^{\mathrm{a}}$ & $0.329^{\mathrm{a}}$ & 50.2 & NS \\
\hline $\mathrm{Mg} / \mathrm{Ca}$ & $0.164^{b}$ & $0.198^{\mathrm{a}}$ & $0.155^{\mathrm{b}}$ & $0.182^{\mathrm{a}}$ & $0.150^{\mathrm{b}}$ & $0.186^{\mathrm{a}}$ & $0.202^{\mathrm{a}}$ & 19.7 & $* *$ \\
\hline $\mathrm{Mg} / \mathrm{S}$ & $3.334^{\mathrm{a}}$ & $2.635^{\mathrm{b}}$ & $2.169^{b}$ & $2.762^{b}$ & $3.255^{\mathrm{a}}$ & $2.297^{\mathrm{b}}$ & $2.977^{\mathrm{a}}$ & 32.9 & ** \\
\hline $\mathrm{Mg} / \mathrm{B}$ & $0.049^{\mathrm{a}}$ & $0.058^{\mathrm{a}}$ & $0.046^{\mathrm{a}}$ & $0.051^{\mathrm{a}}$ & $0.055^{\mathrm{a}}$ & $0.050^{\mathrm{a}}$ & $0.054^{\mathrm{a}}$ & 39.2 & NS \\
\hline $\mathrm{Mg} / \mathrm{Cu}$ & $0.489^{\mathrm{a}}$ & $0.553^{\mathrm{a}}$ & $0.412^{\mathrm{a}}$ & $0.487^{\mathrm{a}}$ & $0.636^{\mathrm{a}}$ & $0.487^{\mathrm{a}}$ & $0.465^{\mathrm{a}}$ & 62.8 & NS \\
\hline $\mathrm{Mg} / \mathrm{Fe}$ & $0.026^{\mathrm{a}}$ & $0.032^{\mathrm{a}}$ & $0.029^{\mathrm{a}}$ & $0.034^{\mathrm{a}}$ & $0.035^{\mathrm{a}}$ & $0.033^{\mathrm{a}}$ & $0.037^{\mathrm{a}}$ & 41.2 & NS \\
\hline $\mathrm{Mg} / \mathrm{Mn}$ & $0.031^{\mathrm{a}}$ & $0.031^{\mathrm{a}}$ & $0.030^{\mathrm{a}}$ & $0.034^{\mathrm{a}}$ & $0.032^{\mathrm{a}}$ & $0.037^{\mathrm{a}}$ & $0.031^{\mathrm{a}}$ & 58.2 & NS \\
\hline $\mathrm{Mg} / \mathrm{Zn}$ & $0.640^{\mathrm{a}}$ & $0.521^{b}$ & $0.479^{b}$ & $0.674^{\mathrm{a}}$ & $0.653^{\mathrm{a}}$ & $0.485^{\mathrm{b}}$ & $0.633^{\mathrm{a}}$ & 32.1 & $* *$ \\
\hline $\mathrm{S} / \mathrm{N}$ & $0.043^{b}$ & $0.049^{b}$ & $0.061^{\mathrm{a}}$ & $0.053^{\mathrm{b}}$ & $0.049^{b}$ & $0.062^{\mathrm{a}}$ & $0.050^{\mathrm{b}}$ & 21.9 & ** \\
\hline $\mathrm{S} / \mathrm{P}$ & $0.981^{\mathrm{b}}$ & $1.098^{\mathrm{b}}$ & $1.230^{\mathrm{a}}$ & $1.130^{b}$ & $1.092^{b}$ & $1.405^{\mathrm{a}}$ & $1.021^{b}$ & 25.8 & $* *$ \\
\hline $\mathrm{S} / \mathrm{K}$ & $0.092^{\mathrm{b}}$ & $0.106^{\mathrm{b}}$ & $0.119^{b}$ & $0.120^{\mathrm{b}}$ & $0.101^{b}$ & $0.148^{\mathrm{a}}$ & $0.109^{b}$ & 27.8 & ** \\
\hline $\mathrm{S} / \mathrm{Ca}$ & $0.053^{b}$ & $0.078^{\mathrm{a}}$ & $0.077^{\mathrm{a}}$ & $0.069^{\mathrm{a}}$ & $0.050^{\mathrm{b}}$ & $0.084^{\mathrm{a}}$ & $0.073^{\mathrm{a}}$ & 26.7 & $* *$ \\
\hline $\mathrm{S} / \mathrm{Mg}$ & $0.328^{\mathrm{b}}$ & $0.401^{b}$ & $0.524^{\mathrm{a}}$ & $0.391^{\mathrm{b}}$ & $0.364^{b}$ & $0.469^{\mathrm{a}}$ & $0.363^{b}$ & 34.7 & ** \\
\hline $\mathrm{S} / \mathrm{B}$ & $0.015^{\mathrm{b}}$ & $0.023^{\mathrm{a}}$ & $0.023^{\mathrm{a}}$ & $0.019^{\mathrm{b}}$ & $0.018^{\mathrm{b}}$ & $0.022^{\mathrm{a}}$ & $0.019^{b}$ & 34.9 & $* *$ \\
\hline $\mathrm{S} / \mathrm{Cu}$ & $0.158^{\mathrm{a}}$ & $0.207^{\mathrm{a}}$ & $0.181^{\mathrm{a}}$ & $0.181^{\mathrm{a}}$ & $0.205^{\mathrm{a}}$ & $0.210^{\mathrm{a}}$ & $0.159^{\mathrm{a}}$ & 55.6 & NS \\
\hline $\mathrm{S} / \mathrm{Fe}$ & $0.008^{b}$ & $0.013^{\mathrm{a}}$ & $0.014^{\mathrm{a}}$ & $0.012^{\mathrm{a}}$ & $0.011^{\mathrm{b}}$ & $0.015^{\mathrm{a}}$ & $0.013^{\mathrm{a}}$ & 39.2 & $* *$ \\
\hline
\end{tabular}




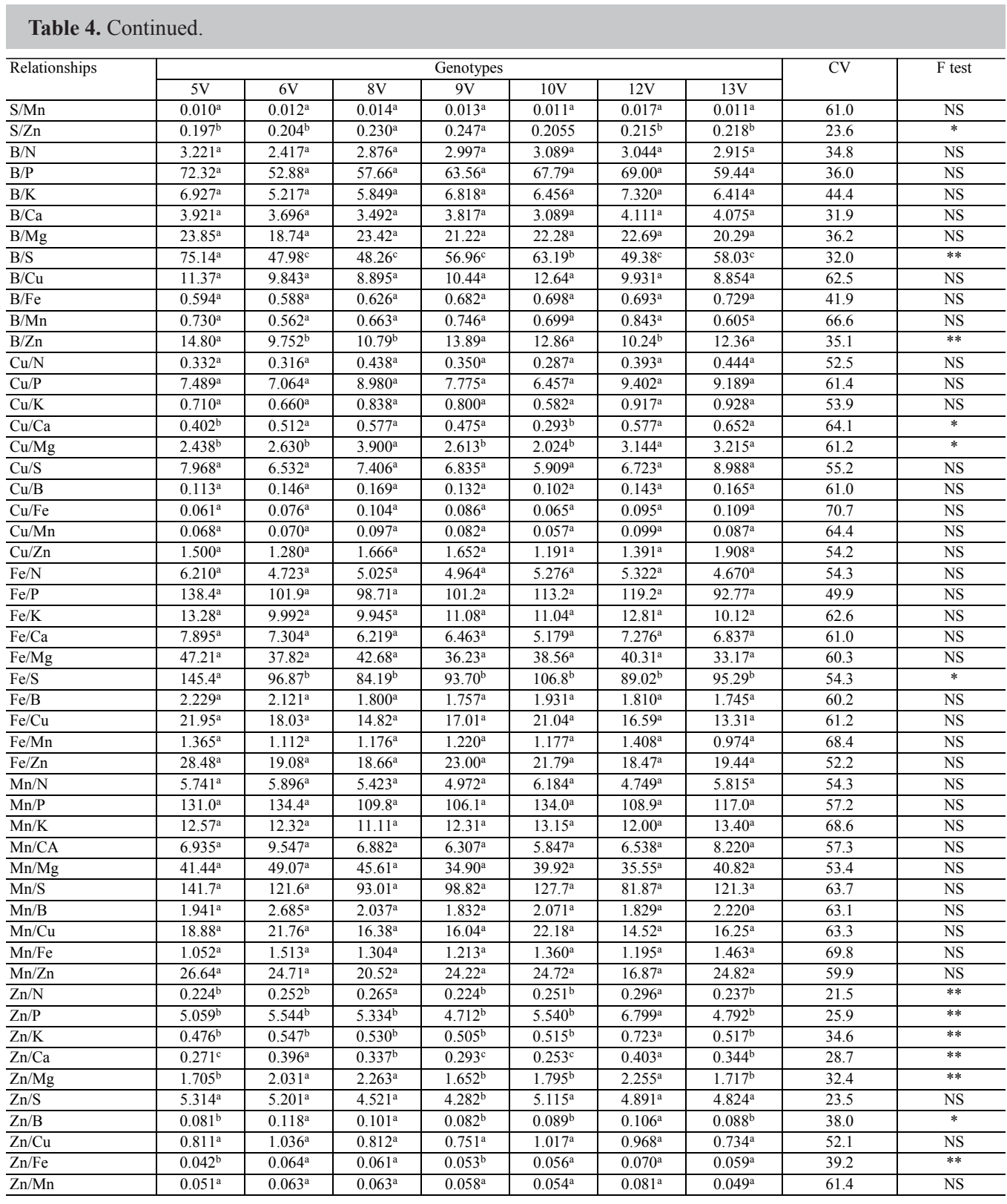

For each relationship between two nutrients, the mean values followed by different letters indicate significant differences between genotypes according to the Scott-Knott test at $5 \%$ probability. NS = not significant; **highly significant $(\mathrm{P} \leq 0.01)$; *significant $(\mathrm{P} \leq 0.05)$.

In general, there was marked variation in the nutrient content of leaves, demonstrating that the nutritional requirement of coffee leaves can vary with both genotype and physiological stage. Changes in the nutrient content of different conilon coffee genotypes were observed months

Genetics and Molecular Research 15 (4): gmr.15048839 
after flowering and have been attributed to nutrient reallocation from the vegetative organs to bean production (Prezotti and Bragança, 2013).

A balanced supply of $\mathrm{N}$ determines $\mathrm{P}$ absorption and translocation rates (Groot et al., 2003). The $8 \mathrm{~V}, 9 \mathrm{~V}$, and $12 \mathrm{~V}$ genotypes exhibited the highest $\mathrm{P}$ and $\mathrm{Cu}$ values. The highest $\mathrm{N}$, $\mathrm{P}$, and $\mathrm{Cu}$ values in those clones can be attributed to the greater need for these nutrients for biochemical reactions, the greater nutrient redistribution in growing points, and an increase in their storage within vacuoles. Under conditions of limited $\mathrm{N}$ and $\mathrm{P}$, greater efficiency of nutrient use may be an important factor, as the plant could produce a greater yield with a lower requirement for these nutrients (Tomaz et al., 2009; Taiz et al., 2015).

C. canephora clones showed greater growth in terms of height, stem diameter, leaf area, and root volume and length when subjected to higher doses of $\mathrm{N}$ and $\mathrm{P}$ (Martins et al., 2013). Covre et al. (2013) also observed variation among C. canephora clones. Notably, the $8 \mathrm{~V}$ conilon genotype showed greater growth compared with other genotypes due to increased absorption of $\mathrm{N}$ and P. Overall, the $8 \mathrm{~V}$ and $12 \mathrm{~V}$ genotypes showed the highest production of biomass due to the balance of nutrients allocated between the shoot and root (Contarato et al., 2010).

Among the 110 LNCRs, 37 were similar $(\mathrm{P} \leq 0.05)$, indicating that $33.6 \%$ of the nutritional indexes were equal among the different genotypes during the pre-flowering stage (Table 3). Greater differences between LNCRs were exhibited by the F-test (ANOVA) and were classified with respect to the nutrient in the numerator: $\mathrm{S}$, nine; $\mathrm{Zn}$, seven; $\mathrm{Ca}$, six; $\mathrm{P}$, four, $\mathrm{N}$ and $\mathrm{Mg}$, three; $\mathrm{B}$ and $\mathrm{Cu}$, two; $\mathrm{K}$ and $\mathrm{Fe}$, one; and $\mathrm{Mn}$, zero relationships (Table 3).

A total of 55 LNCRs were similar $(\mathrm{P} \leq 0.05)$ during the bean-filling stage, indicating that $50 \%$ were significantly different in at least one of the genotypes according to the F-test (Table 4). LNCRs exhibited greater differences according to the F-test (ANOVA) and were classified with respect to the nutrient in the numerator: $\mathrm{S}$, nine; Ca, eight; P, seven; K, six; N, $\mathrm{Mg}, \mathrm{B}, \mathrm{Cu}$ and $\mathrm{Fe}$, four relationships; and $\mathrm{Mg}$, one relationship (Table 4).

In the present study, the influence of genotype on LNCs and LNCRs was clear, but varied according to phenological stage. For example, values for the N/Ca relationship ranged from 1.2 to 1.6 in the $5 \mathrm{~V}$ and $6 \mathrm{~V}$ genotypes, respectively, during the pre-flowering stage, and from 1.2 to 1.9 in the $10 \mathrm{~V}$ and $13 \mathrm{~V}$ genotypes, respectively, during the bean-filling stage. Therefore, the results of the present study show that leaf nutrient standards vary according to both genotype and phenological stage.

Differences among genotypes with respect to both LNCs and LNCRs are probably related to the high genetic variability in conilon coffee, because this species exhibits crosspollination, with marked phenotypic differences among genotypes in terms of plant height, production capacity, leaf shape, fruit ripening, and extraction capacity (Fonseca et al., 2004, 2006; Tomaz et al., 2009; Contarato et al., 2010; Covre et al., 2013; Partelli et al., 2014b; Marré et al., 2015).

Genetic variability may confer greater absorption capacity (mainly related to the root system) of certain nutrients to some genotypes subsequently improving their photosynthetic performance. For example, the $8 \mathrm{~V}, 9 \mathrm{~V}$, and $12 \mathrm{~V}$ genotypes showed higher $\mathrm{N}, \mathrm{P}, \mathrm{K}, \mathrm{Mg}, \mathrm{S}$, and $\mathrm{Cu}$ values, which are essential nutrients for adequate functioning of the photosynthetic apparatus. $\mathrm{N}$ and $\mathrm{Mg}$ are important elements related to ribulose-1,5-bisphosphate carboxylase/ oxygenase (Rubisco), which is a key photosynthesis enzyme (Taiz et al., 2015). P is essential for lipid membranes and ATP synthesis (Marschner, 1995), while S plays key roles in ironsulfur enzyme activity during electron transport and in $\mathrm{CO}_{2}$ incorporation into sugar in the Calvin cycle in chloroplasts (Taiz et al., 2015).

$\mathrm{K}$ participates directly in stomatal opening, osmotic adjustment, photosynthate

Genetics and Molecular Research 15 (4): gmr.15048839 
translocation, and enzyme activation (Taiz et al., 2015). Thus, strengthening of the photosynthetic apparatus can result in higher photosynthate production and growth in the $8 \mathrm{~V}$, $9 \mathrm{~V}$, and $12 \mathrm{~V}$ genotypes, as observed by Covre et al. (2013). However, plant yield is dependent on assimilate partitioning; consequently, the highest photosynthetic rate may not result in the highest yield (Long et al., 2015).

Several studies have shown that different genotypes (Wadt et al., 1999) exhibit differences in nutrient concentrations under cultivation conditions (Partelli et al., 2006) and in different regions (Dara et al., 1992; Wadt and Dias, 2012). Wadt et al. (1999), who studied different Eucalyptus genotypes, found distinct differences in $\mathrm{N}, \mathrm{P}, \mathrm{K}$, and Ca nutrient concentrations among conilon genotypes. Reis Júnior and Monnerat (2003) found that different yields of sugar cane plants were associated with different diagnosis and recommendation integrated system relationships. Those authors also reported that leaf nutrient standards need to be regionalized to ensure clear diagnosis.

The conilon coffee genotypes studied in the present research exhibited different leaf nutrient standards and may therefore differ in their nutritional requirement to produce the same yield and may require genotype-specific diagnosis. Although some genotypes presented only one nutrient with a different mean value, this may indicate that each genotype could have its own leaf nutrient standard.

Our findings showed that the clustering method revealed two consistent clusters, which overall, further support the idea that LNC can vary according to conilon coffee genotype, and although previous studies provided reference levels, it is possible that plants exhibit different nutrient requirements depending on their growth conditions.

In conclusion, two clusters were formed by the clustering method, showing that genetic diversity exists in LNCs and LNCRs among conilon coffee genotypes, during both the pre-flowering and bean-filling stages. Additionally, the $8 \mathrm{~V}, 10 \mathrm{~V}$, and $12 \mathrm{~V}$ genotypes exhibited the highest values for most of the nutrients, especially for $\mathrm{N}, \mathrm{P}$, and $\mathrm{Cu}$. Leaf diagnosis can be genotype-specific and may differ between phenological stages, which can help farmers to reduce the cost of fertilizer and improve their income.

\section{Conflicts of interest}

The authors declare no conflict of interest.

\section{ACKNOWLEDGMENTS}

The authors would like to thank the Cooperativa Agrária dos Cafeicultores de São Gabriel, Cooabriel, for providing the conilon crop area and the Universidade Federal do Espírito Santo, UFES, FAPES, CNPq, and CAPES for financial support.

\section{REFERENCES}

Belan LL, Jesus Jr WC, Souza AF, Zambolim L, et al. (2015). Monitoring of leaf rust in conilon coffee clones to improve fungicide use. Australas. Plant Pathol. 44: 5-12. http://dx.doi.org/10.1007/s13313-014-0310-y

CONAB, Companhia Nacional de Abastecimento. Acompanhamento da Safra Brasileira (2016). Primeiro Levantamento. Janeiro 2016. Available at [http://www.conab.gov.br/OlalaCMS/uploads/arquivos/16 012017 _ $01 \_56$ boletim cafe - janeiro 2016.pdf.]. Accessed March 15, 2016.

Contarato CC, Sobreira FM, Tomaz MA, Jesus Jr WC, et al. (2010). Evaluation of the initial development of Conilon coffee clones (Coffea canephora). Sci. Agrar. 11: 65-71.

Genetics and Molecular Research 15 (4): gmr.15048839 
Covre AM, Partelli FL, Mauri AL and Dias MA (2013). Crescimento e desenvolvimento inicial de genótipos de café Conilon. Rev. Agroambiente 7: 193-202.

Covre AM, Partelli FL, Gontijo I and Zucoloto M (2015). Distribuição do sistema radicular de cafeeiro conilon irrigado e não irrigado. Pesq. Agropec. Bras. 50: 1006-1016. http://dx.doi.org/10.1590/S0100-204X2015001100003

Cruz CD (2013). Programa Genes (Versão Windows): Aplicativos Computacionais em Genética e Estatística. Universidade Federal de Viçosa, Viçosa.

Cruz CD, Carneiro PCS and Regazzi AJ (2014). Modelos Biométricos Aplicados ao Melhoramento Genético. Vol. 2. 3rd edn. Editora UFV, Viçosa.

Dalcomo JM, Vieira HD, Ferreira A, Lima WL, et al. (2015). Evaluation of genetic divergence among clones of conilon coffee after scheduled cycle pruning. Genet. Mol. Res. 14: 15417-15426. http://dx.doi.org/10.4238/2015. November.30.19

Dara ST, Fixen P and Eand Gelderman RH (1992). Sufficiency level and Diagnosis and Recommendation Integrated System approaches for evaluating the nitrogen status of the corn. Agron. J. 84: 1006-1010. http://dx.doi.org/10.2134/ agronj1992.00021962008400060020x

Davis AP, Tosh J, Ruch N and Fay MF (2011). Growing coffee: Psilanthus (Rubiaceae) subsumed on the basis of molecular and morphological data implications for the size, morphology, distribution and evolutionary history of Coffea. Bot. J. Linn. Soc. 167: 357-377. http://dx.doi.org/10.1111/j.1095-8339.2011.01177.x

Davis AP, Gole TW, Baena S and Moat J (2012). The impact of climate change on indigenous Arabica coffee (Coffea arabica): predicting future trends and identifying priorities. PLoS One 7: e47981. http://dx.doi.org/10.1371/journal. pone. 0047981

Esther AK and Adomako B (2010). Genetic and environmental correlations between bean yield and agronomic traits in Coffea canephora. J. Plant Breed. Crop Sci. 4: 64-72.

Favarin JL, Vitti GC, Dourado Neto D, Favarin JL, Junior., et al. (2007). Teor de zinco no café como variável da disponibilidade no solo e extrator. Rev. Bras. Cienc. Solo 31: 1191-1196. http://dx.doi.org/10.1590/S0100$\underline{06832007000500034}$

Ferreira AD, Carvalho GR, Abrahão JCR, Rezende RM, et al. (2013). Dinâmica dos micronutrientes em cafeeiros enxertados. Rev. Ceres 60: 262-269. http://dx.doi.org/10.1590/S0034-737X2013000200016

Fonseca AFA, Ferrão MAG, Ferrão RG, Verdin Filho AC, et al. (2004). Conilon Vitória - Incaper 8142': improved Coffea canephora var. kouillou clone cultivar for the state of Espírito Santo. Crop Breed. Appl. Biotechnol. 4: 503-505. http://dx.doi.org/10.12702/1984-7033.v04n04a20

Fonseca AFA, Sediyama T, Cruz CD, Sakaiyama NS, et al. (2006). Divergência genética em café Conilon. Pesq. Agropec. Bras. 41: 599-605. http://dx.doi.org/10.1590/S0100-204X2006000400008

Groot CC, Marcelis LFM, Van Den Boogaard R, Kaiser WM, et al. (2003). Interaction of nitrogen and phosphorus nutrition in determining growth. Plant Soil 248: 257-268. http://dx.doi.org/10.1023/A:1022323215010

ICO - International Coffee Organization (2016). Trade statistics. Available at [http://www.ico.org/trade statistics.asp] Accessed March 3, 2016.

Leroy T, De Bellis F, Legnate H, Musoli P, et al. (2014). Developing core collections to optimize the management and the exploitation of diversity of the coffee Coffea canephora. Genetica 142: 185-199. http://dx.doi.org/10.1007/s10709$\underline{014-9766-5}$

Long SP, Marshall-Colon A and Zhu X-G (2015). Meeting the global food demand of the future by engineering crop photosynthesis and yield potential. Cell 161: 56-66. http://dx.doi.org/10.1016/j.cell.2015.03.019

Kathurima CW, Kenji GM, Muhoho SM, Boulanger R, et al. (2012). Genetic diversity among commercial coffee varieties, advanced selections and museum collections in Kenya using molecular markers. Int. J. Biodivers. Conser. 4: 39-46. http://dx.doi.org/10.5897/IJBC11.231

Köppen W (1931) Climatologia. México, Fundo de Cultura Econômica.

Mahalanobis PC (1936). On the generalized distance in statistics. Proc. Nat. Inst. Sci. India 2: 49-55.

Marré WB, Partelli FL, Espindula MC, Dias JRM, et al. (2015). Micronutrient accumulation in conilon coffee berries with different maturation cycles. Rev. Bras. Cienc. Solo 39: 1456-1462. http://dx.doi.org/10.1590/01000683rbcs20140649

Mattiello EM, Pereira MG, Zonta E, Mauri J, et al. (2008). Produção de matéria seca, crescimento radicular e absorção de cálcio, fósforo e alumínio por Coffea canephora e Coffea arabica sob influência da atividade do alumínio em solução. Rev. Bras. Cienc. Solo 32: 425-434. http://dx.doi.org/10.1590/S0100-06832008000100040

Martins LD, Tomaz MA, Amaral JFT, Christo LF, et al. (2013). Morphological changes in conilon coffee clones submitted to phosphorus levels. Sci. Plena 9: 1-11. http://dx.doi.org/10.1016/j.plantsci.2012.09.001

Martins LD, Rodrigues WN, Machado LS, Brinate SVB, et al. (2015). Evidence of genetic tolerance to low availability of phosphorus in the soil among genotypes of Coffea canephora. Genet. Mol. Res. 14: 10576-10587. http://dx.doi. org/10.4238/2015.September.8.19

Genetics and Molecular Research 15 (4): gmr.15048839 
Marschner H (1995) Mineral Nutrition of Higher Plants. 2nd ed. Academic Press, London.

Mojena R (1977). Hierarchical grouping method and stopping rules an evaluation. Comput. J. 20: 359-363. http://dx.doi. org/10.1093/comjnl/20.4.359

Partelli FL, Vieira HD, Monnerat PH and Viana AP (2006). Estabelecimento de normas Dris em cafeeiro conilon orgânico ou convencional no estado do Espírito Santo. Rev. Bras. Cienc. Solo 30: 443-451. http://dx.doi.org/10.1590/S0100$\underline{06832006000300006}$

Partelli FL, Viera HD, Carvalho VB and Mourão Filho FAA (2007). Diagnosis and recommendation integrated system norms, sufficiency range, and nutritional evaluation of Arabian coffee in two sampling periods. J. Plant Nutr. 30: 1651-1667. http://dx.doi.org/10.1080/01904160701615525

Partelli FL, Covre AM, Oliveira MG, Alexandre RS, et al. (2014a). Root system distribution and yield of 'Conilon' coffee propagated by seeds or cuttings. Pesq. Agropec. Bras. 49: 349-355. http://dx.doi.org/10.1590/S0100$\underline{204 X 2014000500004}$

Partelli FL, Espindula MC, Marré WB and Vieira HD (2014b). Dry matter and macronutrient accumulation in fruits of conilon coffee with different ripening cycles. Rev. Bras. Cienc. Solo 38: 214-222. http://dx.doi.org/10.1590/S010006832014000100021

Prezotti LC and Bragança SM (2013). Acúmulo de massa seca, N, P e K em diferentes genéticos de café Conilon. Coffee Sci 8: 284-294.

Reis Júnior RA and Martinez HEP (2002). Adição de Zn e absorção, translocação e utilização de Zn e P por cultivares de cafeeiro. Sci. Agric. 59: 537-542. http://dx.doi.org/10.1590/S0103-90162002000300019

Reis Júnior RA and Monnerat PH (2003). Norms establishment of the Diagnosis and Recommendation Integrated System (DRIS) for nutritional diagnosis of sugarcane. Pesq. Agropec. Bras. 38: 277-282. http://dx.doi.org/10.1590/S0100$\underline{204 X 2003000200015}$

Silva FC (2009). Manual de análise química de solos, plantas e fertilizantes. 2nd ed. Embrapa, Brasília, DF.

Silva FAZ and Azevedo CAV (2002). Versão do programa computacional assistat para o sistema operacional Windows. Rev. Bras. Prod. Agroind. 4: 71-78.

Souza FF, Caixeta ET, Ferrão LFV, Guilherme Ferreira Pena GF, et al. (2013). Molecular diversity in Coffea canephora germplasm conserved and cultivated in Brazil. Crop Breed. Appl. Biotechnol. 13: 221-227. http://dx.doi.org/10.1590/ $\underline{\text { S1984-70332013000400001 }}$

Ronchi CP, Sousa Júnior JM, Ameida WL and Souza DS (2015). Morfologia radicular de cultivares de café arábica submetidas a diferentes arranjos espaciais. Pesq. Agropec. Bras. 50: 187-195. http://dx.doi.org/10.1590/S0100$\underline{204 X 2015000300001}$

Taiz L, Zeiger E, Møller IM and Murphy A (2015). Plant Physiology and Development. 6th edn. Sinauer Associates, Sunderland, MA.

Tomaz MA, Martinez HEP, Cruz CD and Freitas RS (2009). Eficiência relacionada à absorção e utilização de Nitrogênio, Fósforo e Enxofre em plantas de cafeeiros enxertados, cultivados em vasos. Cienc. Agrotec. 33: 993-1001.

Wadt PGS and Dias JRM (2012). Normas DRIS regionais e inter-regionais na avaliação nutricional de café Conilon. Pesq. Agropec. Trop. 47: 822-830.

Wadt PGS, Novais RFD, Alvarez VH and Barros NFD (1999). Variations on the nutritional status of eucalypt as influenced by the genetic material and age of tree. Pesq. Agropec. Bras. 34: 1796-1803.

Genetics and Molecular Research 15 (4): gmr.15048839 